compared to $1 / 14$ LGI1-Ab+patients $(\mathrm{p}=0.0024)$ and $1 / 12$ healthy controls.

Conclusion Neuropathic pain may be present in both LGI-Ab + and CASPR2-Ab+patients, and is immunotherapy responsive. Serum IgG from CASPR2-Ab+patients more frequently bound sensory neurons and dorsal root ganglia, suggesting pathophysiological differences which may underlie the more severe pain in these patients.

\section{DISEASE REACTIVATION AFTER CESSATION OF DISEASE-MODIFYING THERAPY IN RELAPSING- REMITTING MULTIPLE SCLEROSIS}

\begin{abstract}
1,2 Izanne Roos, ${ }^{1,2}$ Charles B Malpas, ${ }^{3}$ Emmanuelle Leray, ${ }^{2,4}$ Katherine Buzzard, ${ }^{4,5}$ Olga Skibina, ${ }^{6,7}$ Jeannette Lechner-Scott, ${ }^{8}$ Pamela McCombe, ${ }^{9}$ Mark Slee, ${ }^{10}$ Ernest Butler, ${ }^{11}$ Richard Macdonell, ${ }^{5,12}$ Anneke van der Walt, ${ }^{13}$ Suzanne Hodgkinson, ${ }^{14}$ Michael Barnett, ${ }^{15}$ Steve Vucic, ${ }^{16}$ Sandra Vukusic, ${ }^{5,12}$ Helmut Butzkueven, ${ }^{1,2}$ Tomas Kalincik. ${ }^{1}$ CORe Unit, Department of Medicine, University of Melbourne, Melbourne, VIC, Australia; ${ }^{2}$ Department of Neurology, Royal Melbourne Hospital, Melbourne, VIC, Australia; ${ }^{3}$ Rennes University, Rennes, France; ${ }^{4}$ Department of Neurology, Box Hill Hospital, Monash University, Melbourne, VIC, Australia; ${ }^{5}$ Department of Neurology, The Alfred Hospital, Melbourne, VIC, Australia; ${ }^{6}$ School of Medicine and Public Health, University of Newcastle, Newcastle, NSW, Australia; 'Department of Neurology, John Hunter Hospital, Hunter New England Health, Newcastle, NSW, Australia; ${ }^{8}$ Department of Neurology, Royal Brisbane and Women's

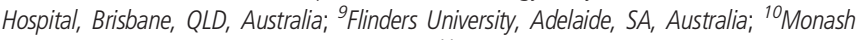
Medical Centre, Melbourne, VIC, Australia; ${ }^{11}$ Department of Neurology, Austin Health, Melbourne, VIC, Australia; ${ }^{12}$ Department of Neuroscience, Central Clinical School, Monash University, Melbourne, VIC, Australia; ${ }^{13}$ Liverpool Hospital, Sydney, NSW, Australia; ${ }^{14}$ Brain and Mind Centre, Sydney, NSW, Australia; ${ }^{15}$ Westmead Hospital, Sydney, NSW, Australia;

${ }^{16}$ Hospital Neurologique Pierre Wertheimer, Lyon, France
\end{abstract}

\subsection{6/bmjno-2021-ANZAN.8}

Objectives To evaluate the rate of return of disease activity after cessation of multiple sclerosis (MS) disease-modifying therapy.

Methods This was a retrospective cohort study from two large observational MS registries: MSBase and OFSEP. Patients with relapsing-remitting MS who had ceased a disease-modifying therapy and were followed up for the subsequent 12-months were included in the analysis. The primary study outcome was annualised relapse rate in the 12 months after disease-modifying therapy discontinuation stratified by patients who did, and did not, commence a subsequent therapy. The secondary endpoint was the predictors of first relapse and disability accumulation after treatment discontinuation.

Results 18029 eligible treatment discontinuation epochs were identified for seven therapies. Rates of relapse started to increase 2-months after natalizumab cessation. Commencement of a subsequent therapy within 2-4 months reduced the magnitude of disease reactivation. After discontinuation of fingolimod, rates of relapse increased overall, and stabilised faster in patients who started a new therapy within 1-2 months. Magnitude of disease reactivation for other therapies was low, but reduced further by commencement of another treatment 1-10 months after treatment discontinuation. Predictors of relapse were higher relapse rate in the year before cessation, female sex, younger age and higher EDSS. Commencement of a subsequent therapy reduced both the risk of relapse (HR 0.76, CI $0.72-0.81)$ and disability accumulation $(0.73,0.65-0.80)$.

Conclusion Understanding the rate of disease reactivation after discontinuing different MS immunotherapies will help guide optimal wash-out times for therapeutic agents during treatment sequencing.

\section{9 PREDICTING INFECTION RISK IN MULTIPLE SCLEROSIS PATIENTS TREATED WITH OCRELIZUMAB: A RETROSPECTIVE COHORT STUDY}

${ }^{1}$ Nabil Seery, ${ }^{2,3}$ Sifat Sharmin, ${ }^{1,4}$ Vivien Li, 1,2,3Ai-Lan Nguyen, ${ }^{1}$ Claire Meaton, ${ }^{1}$ Roberts Atvars, ${ }^{5}$ Nicola Taylor, ${ }^{5}$ Kelsey Tunnell, ${ }^{5}$ Joh Carey, ${ }^{1,6}$ Mark P Marriott, ${ }^{1,6}$ Katherine A Buzzard, 1,2,3 Izanne Ross, ${ }^{1,4}$ Chris Dwyer, ${ }^{1}$ Josephine Baker, ${ }^{1}$ Lisa Taylor, ${ }^{2,7}$ Kymble Springs, ${ }^{1,4}$ Trevor J Kilpatrick, ${ }^{1,2,3}$ Tomas Kalincik, ${ }^{1,8,9}$ Mastura Monif. ${ }^{1}$ Melbourne MS Centre, Department of Neurology, The Royal Melbourne Hospital, Parkville, VIC, Australia; ${ }^{2}$ Department of Medicine, The University of Melbourne, Melbourne, VIC, Australia; ${ }^{3}$ Clinical Outcomes Research Unit, The University of Melbourne, Melbourne, VIC, Australia; ${ }^{4}$ Florey Institute of Neurosciences and Mental Health, The University of Melbourne, Parkville, VIC, Australia; ${ }^{5}$ Day Medical Centre, Royal Melbourne Hospital, Parkville, Melbourne, VIC, Australia; ${ }^{6}$ Department of Neuroscience, Eastern Health Clinical School, Box Hill, VIC, Australia; 'Department of Immunology, The Royal Melbourne Hospital, Melbourne, VIC, Australia; ${ }^{8}$ MS and Neuroimmunology Department, Alfred Health, Melbourne, VIC, Australia; ${ }^{9}$ Department of Neuroscience, Monash University, Melbourne, VIC, Australia

\subsection{6/bmjno-2021-ANZAN.9}

Objective To examine factors determining risk of self-reported infections and antimicrobial use in patients receiving Ocrelizumab for MS.

Methods Retrospective, observational cohort study conducted in Ocrelizumab-treated patients at the Royal Melbourne Hospital. The association of clinical and laboratory factors with self-reported infection rate and antimicrobial use were estimated using univariate and multivariable logistic regression models.

Results 185 patients were included in the study, and 176 infections were reported in 89 patients (46.1\%), and in 47 patients $(25.3 \%)$ antimicrobial use was identified. In univariate analyses, a higher serum IgA was associated with reduced odds of infection (OR 0.44 , 95\% CI 0.25 - 0.76). In multivariable analyses, older age (OR $0.94,95 \%$ CI 0.88 - 0.99), higher serum IgA (OR 0.37, 95\% CI 0.17 - 0.80) and higher serum IgG (OR 0.81, 95\% CI 0.67 - 0.99) were associated with reduced odds of infection. Older age (OR 0.85, 95\% CI 0.75 - 0.96) and higher serum IgA (OR 0.23, 95\% CI 0.07 0.79 ) were associated with reduced odds of antimicrobial use, whilst longer MS disease duration (OR 1.22, 95\% CI 1.06 1.41) and higher EDSS (OR 1.99, 95\% CI 1.02 - 3.86) were associated with increased odds of antimicrobial use.

Conclusions Higher serum IgA, IgG and older age were associated with reduced odds of infection. Our findings highlight non-uniformity of infection risk in Ocrelizumab-treated MS patients, and substantiate the need to monitor immunoglobulin levels pre-treatment and whilst on therapy.

\section{REAL-WORLD EXPERIENCE WITH OCRELIZUMAB IN THE MSBASE REGISTRY - AUSTRALIAN RRMS COHORT}

\begin{abstract}
${ }^{1,2}$ Helmut Butzkueven, ${ }^{2}$ Tim Spelman, ${ }^{3}$ Tomas Kalincik, ${ }^{4}$ Katherine Buzzard, ${ }^{1}$ Anneke Van der Walt, ${ }^{5}$ Jeanette Lechner-Scott, ${ }^{6}$ Suzanne Hodgkinson, ${ }^{7}$ Ernest Butler, ${ }^{8}$ Richard Macdonell, ${ }^{9}$ Mark Slee, ${ }^{2}$ MSBase Study Group, ${ }^{10}$ Bruno Marcel. ${ }^{1}$ Monash University, Melbourne, VIC, Australia; ${ }^{2}$ MSBase Foundation, Melbourne, VIC, Australia; ${ }^{3}$ University of Melbourne, Melbourne, VIC, Australia; ${ }^{4}$ Box Hill Hospital, Box Hill, VIC, Australia; ${ }^{5}$ University of Newcastle, Newcastle, NSW, Australia; ${ }^{6}$ Liverpool Hospital, Sydney, NSW, Australia; ${ }^{7}$ Monash Medical Centre, Melbourne, VIC, Australia; ${ }^{8}$ Austin Health, Melbourne, VIC, Australia; ${ }^{9}$ Flinders University, Adelaide, SA, Australia; ${ }^{10}$ Roche Products Pty Ltd, Sydney, NSW, Australia
\end{abstract}

\subsection{6/bmjno-2021-ANZAN.10}

Introduction Ocrelizumab (OCR) is a humanised anti-CD $20^{+}$ monoclonal antibody for the treatment of Multiple Sclerosis. 
Objectives In Australian MSBase clinics, we describe baseline characteristics of relapsing-remitting MS (RRMS) patients treated with OCR, treatment pathways and early clinical outcomes.

Methods Secondary analysis using MSBase Registry data for RRMS patients with OCR initiation within 3 months of MSBase recorded visit. Descriptive statistics included demographics, disease course/duration, prior disease modifying therapies (DMT) and EDSS. Relapse data was described in patients with $\geq 6$ months follow-up.

Results As of 4 June 2020, MSBase included 624 eligible Australian RRMS patients newly treated with OCR. Median age at OCR initiation was 42.5 years. OCR was first line therapy in $18.9 \%$ of patients. Most frequent DMT's in the 12 months prior to OCR were natalizumab (32.1\%) and fingolimod (24.8\%). Of 434 RRMS patients with $\geq 6$ months follow-up, 392 remained relapse free $(90.3 \%$; 95\% CI 81.6, 99.7) over a mean OCR exposure of 1.35 years. In this group, the annualized relapse rate (ARR) was 0.10 (95\% CI $0.08-0.13$ ), compared to an ARR of 0.83 in the 24 months pre-OCR start. Treatment discontinuation was recorded for 20 of these 434 patients In the overall RRMS cohort, treatment persistence at 12 and 24 months was 94.3\% (95\%CI: 90.9\%96.1\%\%) and $88.7 \%$ (95\%CI 77.2\%-94.0\%), respectively.

Conclusion Almost 20\% of RRMS patients treated with OCR in Australian MSBase centres received OCR as a first line treatment. During OCR treatment, relapses and OCR discontinuations were rare.

\section{WORSENING LONGITUDINAL REACTION TIME TRAJECTORIES USING THE MSREACTOR COMPUTERISED BATTERY PREDICTS CONFIRMED EDSS PROGRESSION}

${ }^{1}$ Daniel Merlo, ${ }^{1} \mathrm{Jim}$ Stankovich, ${ }^{2}$ Claire Bai, ${ }^{2}$ Tomas Kalincik, ${ }^{1}$ Melissa Gresle, ${ }^{3}$ Jeannette Lechner-Scott, ${ }^{4}$ Trevor Kilpatrick, ${ }^{5}$ Michael Barnett, ${ }^{6}$ Bruce Taylor, ${ }^{4}$ David Darby, ${ }^{1}$ Helmut Butzkueven, ${ }^{1}$ Anneke van der Walt. ${ }^{1}$ MSNI, Central Clinical School, Monash University, Melbourne, VIC, Australia; ${ }^{2}$ CORe, Department of Medicine at RMH, University of Melbourne, Melbourne, VIC, Australia; ${ }^{3}$ Department of Neurology, John Hunter Hospital, Newcastle, NSW, Australia; ${ }^{4}$ Florey Institute of Neuroscience and Mental Health, Melbourne, VIC, Australia; ${ }^{5}$ Brain and Mind Centre, Sydney, NSW, Australia; ${ }^{6}$ Department of Neurology, Royal Hobart Hospital, Hobart, TAS, Australia

\subsection{6/bmjno-2021-ANZAN.11}

Objectives To identify and validate longitudinal reaction time trajectories in relapsing remitting multiple sclerosis using a computerised cognitive battery and latent class mixed modelling, and to assess the association between reaction time trajectories and disability progression.

Methods Participants serially completed web-based computerised reaction time tasks measuring psychomotor speed, visual attention and working memory. Testing sessions were completed 6-monthly with the option of additional home based testing. Participants who completed at least three testing sessions over a minimum of 180 days were included in the analysis. Longitudinal reaction times were modelled using Latent Class Mixed Models to group individuals sharing similar latent characteristics. Models were tested for consistency using a cross-validation approach. Inter-class differences in the probability of reaction time worsening and the probability of 6month confirmed disability progression were assessed using survival analysis.

Results A total of 460 relapsing remitting multiple sclerosis patients were included. For each task of the MSReactor computerised cognitive battery, the optimal model comprised of 3 latent classes. All tasks could identify a group with high probability of reaction time slowing. The visual attention and working memory tasks could identify a group of participants who were 3.7 and 2.6 times more likely to experience a 6-month confirmed disability progression, respectively. Participants could be classified into predicted cognitive trajectories after just 5 tests with between $64 \%$ and 89\% accuracy.

Conclusion Latent class modelling of longitudinal cognitive data collected by the MSReactor battery identified a group of patients with worsening reaction times and increased risk of disability progression.

\section{VOLUMETRIC AND CONNECTIVITY PROFILE OF REGIONAL THALAMIC ABNORMALITY IN AMYOTROPHIC LATERAL SCLEROSIS}

${ }^{1}$ Sicong Tu, ${ }^{2}$ Marion Sourty, ${ }^{1}$ Fernando Calamante, ${ }^{3}$ Manoj Saranathan, ${ }^{4}$ Ricarda Menke, ${ }^{4}$ Kevin Talbot, ${ }^{1}$ Matthew Kiernan, ${ }^{4}$ Martin Turner. ${ }^{1}$ Brain and Mind Centre, University of Sydney, Sydney, NSW, Australia; ${ }^{2}$ Université de Strasbourg, Strasbourg, France; ${ }^{3}$ University of Arizona, Tucson, Arizona, USA; ${ }^{4}$ Nuffield Department of Clinical Neurosciences, University of Oxford, Oxford, UK

\subsection{6/bmjno-2021-ANZAN.12}

Objectives Neurodegeneration in ALS follows a diffuse pattern of cortical involvement. ${ }^{1}$ We have previously highlighted that thalamic abnormality is a robust disease signature in $\mathrm{ALS},{ }^{2}$ but the integrity of thalamic nuclei and their clinical association remains unclear. We employed a novel segmentation technique for thalamic nuclei and track-weighted functional connectivity (TW-sFC) to characterize volumetric and connectivity profiles of regional thalamic abnormality.

Methods Forty ALS patients and 27 age-and-education matched controls were recruited. All patients underwent comprehensive clinical examination and 3T MRI scan (T1; DWI; rs-fMRI). Thalamic nuclei were robustly segmented from T1 images using the THOMAS pipeline. ${ }^{3}$ Whole-brain white matter fibre tracking was performed using MRtrix and combined with resting-state fMRI to generate combined structural and functional connectivity maps (TW-sFC). ${ }^{4}$

Results Reduced thalamus volume was observed bilaterally in ALS compared to control ( $\mathrm{p}$ values < 0.036). Bilateral volumetric reduction was consistently observed across all regions except for the anterior thalamus in ALS ( $p$ values $<0.05$ ). Significant increased TW-sFC was observed in ALS in the right anterior thalamus $(\mathrm{p}=0.03)$ and right anterior ventral nuclei ( $\mathrm{p}<0.01$ ). TW-sFC of the mediodorsal nuclei correlated with disease duration $(\mathrm{p}<0.02)$ and disease progression rate $(\mathrm{p}<0.03)$.

Conclusions Regional thalamic abnormalities are present in ALS and hold a significant association with clinical features. Variability in thalamic connectivity demonstrated significant clinical associations with disease duration, progression rate, and upper motor dysfunction. The findings reinforce that diffusion and functional MR imaging modalities are promising markers of disease burden in ALS.

\section{REFERENCES}

1. Brettschneider J, Del Tredici K, Toledo J, et al. Stages of pTDP-43 pathology in amyotrophic lateral sclerosis. Ann Neurol 2013;74:20-38.

2. Tu S, Menke R, Talbot K, Kiernan M, Turner M. Regional thalamic MRI as a marker of widespread cortical pathology and progressive frontotemporal involvement in amyotrophic lateral sclerosis. JNNP 2018;89:1250-1258. 